\title{
Design of Prosthetic Arm
}

\author{
R. Swetha ${ }^{\mathrm{a}, 1}$ and S. NirmalaDevi ${ }^{\mathrm{b}}$ \\ ${ }^{a}$ Student, Dept of Medical Electronics \\ ${ }^{b}$ Professor, Dept of ECE, Centre for Medical Electronics, \\ Anna University Campus, Chennai, India
}

\begin{abstract}
This paper proposes the design of the prosthetic arm by reconstructing the structure and proportions of an amputated arm using high precision methods and dimensions. To achieve this, CT images of the patient's amputated and nonamputated arm are collected from the Rehabilitation centre. The patient CT data were imported to a 3D modelling software i.e., Mimics Innovation Suite version 22.0 Materialise 3-Matic version 14.0 original licensed software. The exported file is given to the Computer-Aided Design software, the geometry of the socket and the prosthetic arm were designed according to the mirrored geometry of the nonaffected arm. 3D rendering for various degrees of movement has been carried out for animation.
\end{abstract}

Keywords: CT images, Thresholding, 3D part calculation, CAD design, Animation.

\section{Introduction}

For humans, the hand is a very important interface. Many interactions take place manually, including the manipulation of objects, interactions, and many other daily tasks [11]. When a human loses his or her limbs, these may be replaced by biomechanical devices such as orthotics and prosthetics [2]. In biomechanics, there is a variation between orthotics and prosthetics. The prosthesis is an artificial device that replaces a missing portion of the body that may be removed due to trauma, illness, or congenital disorders. Orthotics is a device used to help the limbs, or skeletal parts that are weak, ineffective, deformed or hurt. In the traumatic and sudden loss of the upper limb, the psychological effect is often even more intense [7].

It has been made physically more obvious by the upper limb deficiency and the limb presentation is also esthetically uncomfortable because of trauma impact. A prosthesis hand will restore some of a lost limb's positions and assist users with their everyday work [12]. The goal is to make sure that prosthesis and orthotics systems are human-centered and adaptable to the personal and environmental needs of any customer [9]. Measuring customer satisfaction with the prosthesis of the upper limbs and listing design goals for future innovations [8]. A voluntary, self-administered survey has gathered knowledge on the demographics, history, and aims of the prosthesis, comfort, and design goals for the participants. The human control mode is one feature of prosthesis control that makes it exceptional relative to traditional. 
The prosthesis is an artificial extension that substitutes a missing portion of the body such as the upper or lower extremity of the body. An artificial limb is a form of prosthesis that replaces a missing limb, such as arms or legs. The type of artificial limb is selected based on the degree of the amputation or loss and the location of the missing portion of the body. There are two major types of artificial upper extremities. This human-machine systems. Although the user communicates with the computer with the hands or feet in common to all human, this is not the case with most human-prosthetic systems [5]. However, it is uncommon to see commercial use of hand movements in computer-aided sketching and modeling [1].involves Transradial and Trans Humeral Prosthesis [5]Trans radial prosthesis is used to replace the arm that is absent below the elbow. In the prosthetics industry, the trans-radial prosthetic arm is sometimes referred to as the "BE" or the prosthesis below the elbow. An artificial extremity that covers a missing arm above the elbow is a trans humeral prosthesis. The difficulty of the elbow movement leads to trans humeral amputees. A trans humeral prosthetic arm is sometimes referred to as an "AE" or below elbow prosthesis in prosthetics industry [5].

The proposed work involves the design of the prosthetic arm focusing on sketching and $3 \mathrm{D}$ modelling tasks using CAD software. The CT images of the amputated and non-amputated arm were taken in order to determinethe dimensions of the prosthetic arm [6]. The segmentation of both arms is carried out using the thresholding technique by using Mimics software [4]. The computer-aided design of the arm and socket was developed by measuring the arm following the thresholding technique. 3D volume and surface rendering were done to establish the prosthetic movement arm [13-17].

\section{Methodology}

\subsection{Block Diagram}

The block diagram of the overall project which is illustrated in Fig. 1

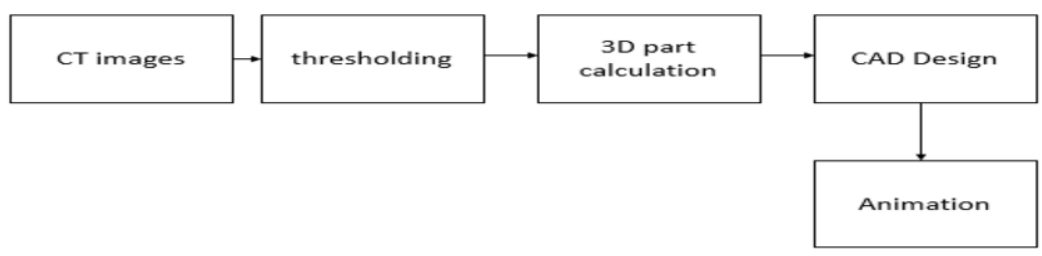

Figure 1. Block diagram of the overall project

\subsection{BlockDiagram Description}

The CT images of the patient's amputated and non-amputated arm are obtained from the Rehabilitation The obtained CT images of were in DICOM Format were loaded in the MIMICS software for segmentation by using the threshold method as shown in Figure 1. After segmentation, a 3D model of the arm is calculated in Mimics software. By using the dimension of the arm measured in Mimics software, the computer-aided 
model of socket and arm is designed. Then for the designed arm animation were created.

\subsection{CTImages}

Computed tomography (CT) scanning of the arm is an imaging technique that uses $\mathrm{x}$ rays to obtain cross-section images of the arm [3]. Medical images from CT scanners consist of grayscale material. With the advent of multidetector CT, the collection of volume has become commonplace and easy to obtain. The benefit of obtaining CT volume acquisition is that it can be reconstructed in three distinct planes: axial, sagittal, and coronal. Fig. 2 and represents the CT images of non-amputated and amputated arm.

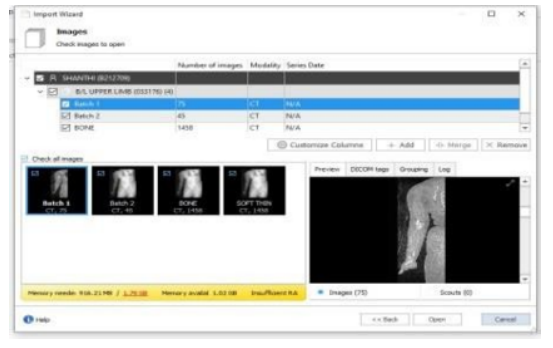

Figure 2. CT image of non-amputated arm

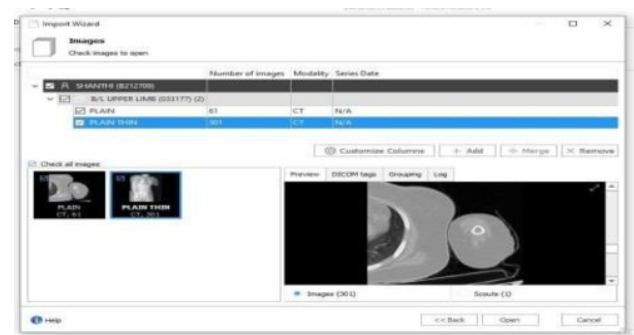

Figure 3. CT image of amputated arm

\subsection{Mimics Software}

Materialize Mimics is an image processing software used for 3D design and modeling. Mimics is an acronym for the Materialize Interactive Medical Image Control System Materialize, Mimics is used to construct 3D surface projections from stacks of 2D image data. These 3D models used for a variety of engineering applications. Fig. 5 represents the CT images of and non-amputated arm in mimics.

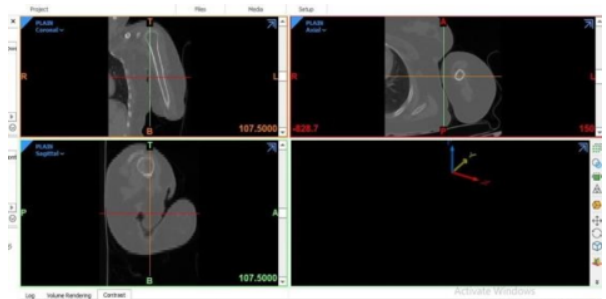

Figure 4.CT Image of Amputated Arm

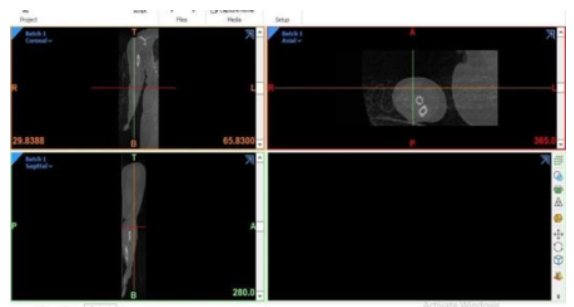

Figure 5.CT Image of Non-Amputated Arm

\section{Results}

\subsection{Segmentation Results}

Patient amputation and non-amputation arm CT images were collected. The CT images have been imported into the Mimics software for segmentation process. The segmentation process covers thresholds. The following Fig. 6 and 7 shows the image of CT images after importing into the Mimics. 


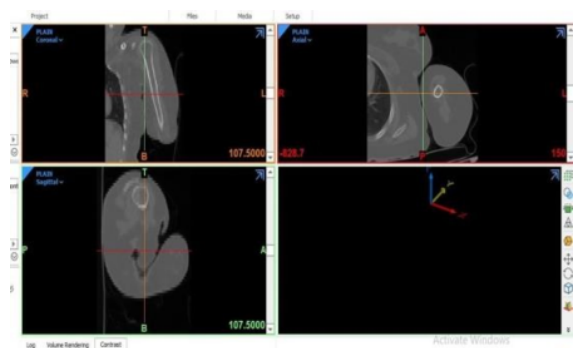

Figure 6. CT Image of Amputated Arm

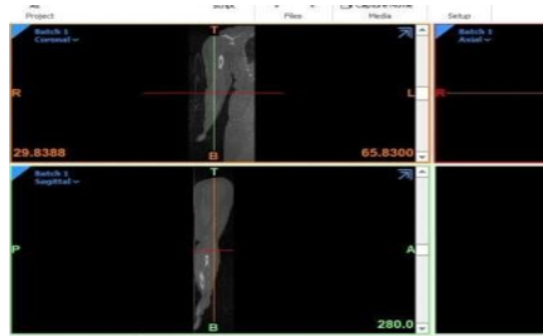

Figure 7. CT Image of Non-Amputated Arm

\subsection{Thresholding}

In a certain Hounsfield range, all pixels are classified as being of the same color or mask. The threshold of non-amputated arm soft tissue is performed between -499 HU and $248 \mathrm{HU}$. Fig. 8 shows the 3D soft tissue of amputated arm after thresholding. Thethresholding of soft tissue of amputated arm is performed with the range of $38 \mathrm{HU}$ to $1025 \mathrm{HU}$. Figure 9 shows the 3D soft tissue of non-amputated arm after thresholding.

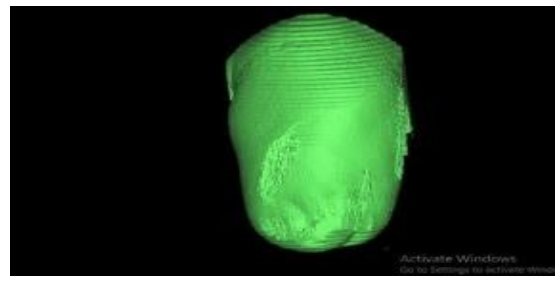

Figure 8. Soft Tissue of Amputated Arm

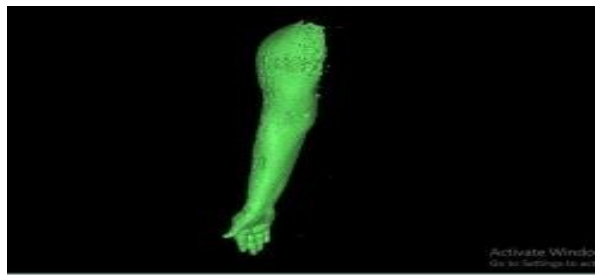

Figure 9. Soft Tissue of Non-Amputated Arm

\subsection{Measurement of AmputatedArm}

Area will be measured for the amputated arm at various lengths. The measurement of amputated arm for length $261.73 \mathrm{~mm}$ is shown in Fig 10. Amputated arm with socket design in shown in Fig. 11.
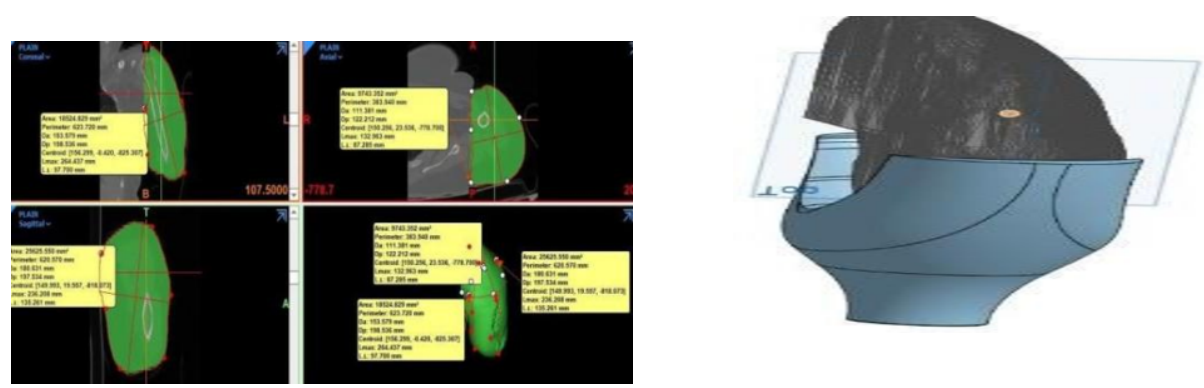

Figure 10. Area Measurement of Amputated Arm at Length $261.73 \mathrm{~mm}$

Figure 11. Amputated Arm with Socket 


\subsection{Design of socket}

The socket is designed based on the amputated arm measurement. The length of amputated arm is $261.73 \mathrm{~mm}$ and the length of non-amputated arm is $291.81 \mathrm{~mm}$, the missing part is $30.08 \mathrm{~mm}$. The socket was designed for the length of $180 \mathrm{~mm}$ where the stump will be fixed in the socket is for $110 \mathrm{~mm}$. The following Fig. 11 show that the designed socket with the amputated arm.

\subsection{Design of forearm}

The forearm is designed on the basis of non-amputated arm dimensions. The nonamputated arm is $216.25 \mathrm{~mm}$ in length. The area measurement of non-amputated arm for length $216.25 \mathrm{~mm}$ is given in the following Fig. 12. The design of the forearm is given in various views, the following figure 13 shows the front view of the forearm.

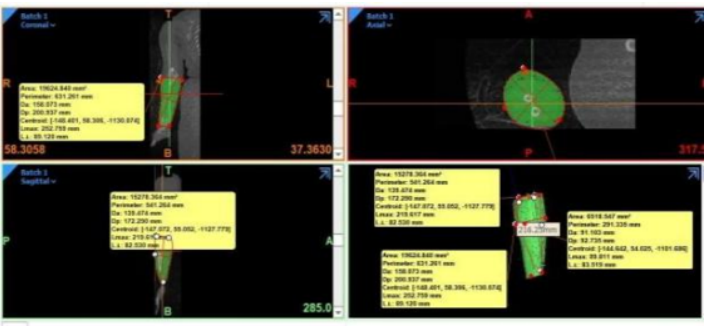

Figure 12. Area Measurement of Non-Amputated Arm at Length $261.25 \mathrm{~mm}$.

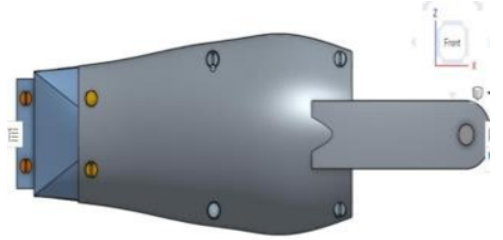

Figure 13. Front view of Forearm

\subsection{Design off hand}

The Hand is designed with the reference to the non-amputated hand. The hand consists of wrist, palm and fingers. The following Fig. 14 is given as the measurement of non-amputated hand. The following Figure 15 is given as design of palm hand (both front and back side)

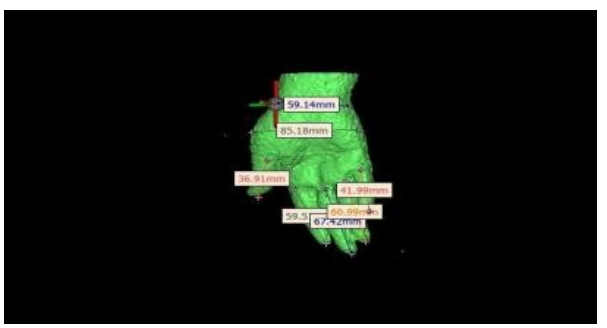

Figure 14. Measurement of Non-Amputated Hand

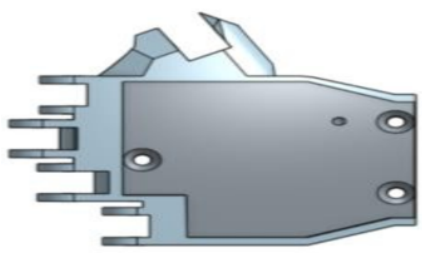

Figure 15. Design of Palm Hand 


\subsection{Design of finger}

The finger consists of 4 parts they are plate, pin, upper part and lower part. The following Fig. 16 is given as design of finger. The following Fig. 17 and Fig. 18 consist of hand design and full arm design.

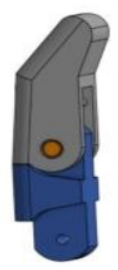

Figure 16. Design of Finger
Figure 17. Design of Hand

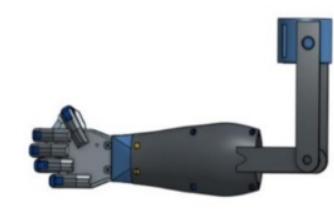

Figure 18. Design of Full arm

\subsection{D Rendering and Animation}

Flexion and Extension for the designed arm is shown in Fig. 20. Based on this design, $3 \mathrm{D}$ surface and volume rendering are carried out as and shown in Fig. 21.

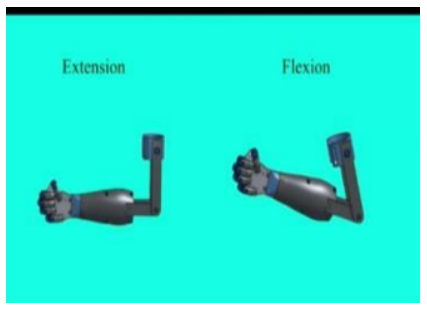

Figure 20. Extension and Flexion of designed arm

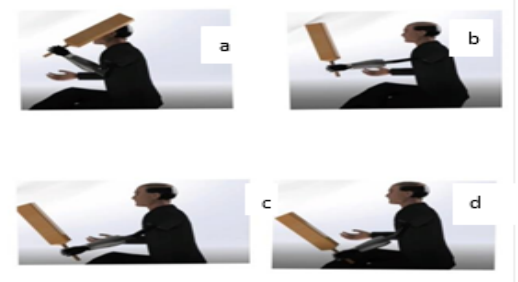

Figure 21. 3D surface and volume rendering at (a) $115^{\circ}$ (b) $90^{\circ}$ (c) $75^{\circ}$ (d) $45^{\circ}$

\section{Conclusion and Future plan}

\subsection{Conclusion}

The CT images were collected for amputated and non-amputated images. Both arms were segmented by the thresholding process in Mimics software. After segmentation in Mimics software, the 3D Arm model was developed. The area measurement of the amputated arm was performed after calculating the 3D part in Mimics software. The CAD model of socket, forearm, and hand was designed by using the dimension of the amputated arm measured from Mimics software. For the designed arm animation model was created using $3 \mathrm{D}$ rendering. 


\subsection{FuturePlan}

The future work would include the 3D printing of arm and FEA analysis. Further attributes such as material selection, FEA analysis of this arm will be taken for further study and the functionality of the arm were achieved by the 3Dparts. AI based VR model can be implemented for future plan.

\section{References}

[1] Aditya Thakur, Rahul Rai. User Study of Hand Gestures for Gesture Based 3d Cad Modelling. International Design Engineering Technical Conferences \& Computers and Information in Engineering Conference (IDETC/CIE).January 19, 2016, DETC2015-46086, V01BT02A017.

[2] Chandra Mohan M, Purushothaman M. Design And Fabrication Of Prosthetic Human Hand Using EEG And Force Sensor With Arduino Micro Controller. Third International Conference On Science Technology Engineering And Management.2017. INSPEC Accession Number: 17507412, Volume 7 Issue No.5.

[3] Disler Dg, Rosenthal. Accuracy of Volume Measurements of Computed Tomography and Magnetic Resonance Imaging Phantoms by Three-Dimensional Reconstruction and Preliminary Clinical Application. 01 Aug 1994, Europe PMC, PMID: 7960623.

[4] D.S. Shin, S. Lee, H.S. Park, S.-B. Lee, M.S. Chung. Segmentation and Surface Reconstruction of a Cadaver Heart on Mimics Software. Via Medica,8 May 2014, FoliaMorphol 2015; 74, 3: 372-377).

[5] Dudley S. Childress. Upper-Limb Prosthetics: Control of Limb Prostheses, Chapter 6D - Atlas of Limb Prosthetics: Surgical, Prosthetic, and Rehabilitation Principles, 1992,oandplibrary, Digital and Resource foundation for the orthotics and prosthetics Community.

[6] John-John Cabibihan, M. KhaleelAbubasha .A Method For 3-D Printing Patient-Specific Prosthetic Arms with High Accuracy Shape and Size. IEEE International Conference on Rehabilitation,2018, Volume: 6, INSPEC Accession Number: 17773708.

[7] John-John Cabibihan, StéphanePattofatto .Towards Humanlike Social Touch for Sociable Robotics and Prosthetics: Comparisons on The Compliance. Conformance and Hysteresis of Synthetic and Human Fingertip Skins, , 2008, Springer, article s12369-008-0008-9.

[8] MidhunVarghes. Model of Prosthetic Arm Finger Using Neural Network. IEEE International Conference on Cyborg and Bionic Systems. 2018. DOI: 10.1109/CBS.2018.8612223.

[9] WHO Standards for Prosthetics and Orthotics. World Health Organization. Geneva. Switzerland. 2017

[10] TeppakornSittiwanchai, Ippei Nakayama, Shi, Nichi Inoue and Jun Kobayash, Trans humeral Prosthesis Prototype With 3d Printing And SEMG-Based Elbow Joint Control Method, Proceedings of The International Conference on Advanced Mechatronic Systems, Kumamoto. 2014, DOI: 10.1109/ICAMechS.2014.6911655.

[11] Taehyun Rhee, J.P. Lewis, Human Hand Modelling from Surface Anatomy, 2010, Researchgate publication $/ 220792173$.

[12] J. Kate, G. Smit, and P. Breedveld, 3D-printed upper limb prostheses: A review, Disab. Rehabil., Assistive Technol., vol. 12, no. 3, pp. 300-314,2017.

[13] Ambeth Kumar, S. Sharmila, Abhishek Kumar, A. K. Bashir, Mamoon Rashid, Sachin Kumar Gupta $\&$ Waleed S. Alnumay .A novel solution for finding postpartum haemorrhage using fuzzy neural techniques. Neural Computing and Applications (2021) (https://doi.org/10.1007/s00521-020-05683-z)

[14] Navadeepika K.M.R., Ambeth Kumar V.D. (2021) Enhancement in Braille Systems-A Survey. Advances in Intelligent Systems and Computing. vol 1257. Springer, Singapore. https://doi.org/10.1007/978-981-15-7907-3_10.

[15] V.D.Ambeth Kumar (2017).Automation of Image Categorization with Most Relevant Negatives. Pattern Recognition and Image Analysis, Vol. 27, No. 3, pp. 371-379, 2017- Springer

[16] M.Ramakrishan et.al, .Enhancement in Footprint Image using Diverse Filtering Technique. in the month of March for the Procedia Engineering journal (Elsevier) Journal Volume 8, No.12, 1072-1080, March 2012 and the paper was published . [doi:10.1016/j.proeng.2012.01.965]

[17] V Rajamani, S Murugavalli . A High Speed Parrallel Fuzzy C-means algorithm for Tumor Segmentation. ICGST International Journal on BIME 6 (1), 2006

[18] K. Sabarinathan et.al ., " Machine Maintenance Using Augmented Reality”, 3rd International Conference on Communication and Electronics Systems (ICCES), 2018. (DOI: 10.1109/CESYS.2018.8723900) 
[19] B. Aravindh; V.D.Ambeth Kumar; G. Harish; V. Siddartth, " A novel graphical authentication system for secure banking systems”, IEEE (ICSTM), Pages: $177-183$, 2-4 Aug. 2017, DOI: 10.1109/ICSTM.2017.8089147 